\title{
Alzheimer and markers
}

\begin{abstract}
The pathophysiological process of Alzheimer's disease (AD) begins long before the diagnosis of clinical dementia. Several biomarkers related to This Have Been Proposed process and new ones are being investigated. The main project objective is this is twofold: 1 ) to Investigate the Ability of cognitive, functional, neuroimaging and metabolomics volatile breath markers to differentiate Differentiates Between Normal aging (NA), mild cognitive impairment (MCI) and early $\mathrm{AD}$, and 2) to determine Whether in Original markers can predict progression to dementia in the MCI group.
\end{abstract}

Keywords: Alzheimer's disease, Neurology, Marker. Biomarker, Early noninvasive metabolomics, Proteomics, Genomics, Transcriptómic, Disability, Positron emission tomography, Amyloid imaging, Cognitive dependence prediction, High complexity foundation
Volume 2 Issue 2 - 2015

\section{De Lema Bruno,' Adjounian Haroution,' lonescu Radu² \\ 'High Complexity Foundation, Pereira, Colombia \\ ${ }^{2}$ University Rovira IVirgili, Tarragona, Espana}

Correspondence: De Lema Bruno, High Complexity Foundation, Pereira, Colombia, Email delemalarre@gmail.com

Received: October 10,2014 | Published: March 12, 2015
Abbreviations: ALZ, Alzheimer; CSF, Cerebrospinal Fluid; PET, Positron Emission Tomography; 18F-FDG, 18F-Fluorodeoxyglucose; MRI, Magnetic Resonance Imaging; MCI, Mild Cognitive Impairment; aMCI, amnestic MCI; NA, Normal Aging; WM, White Matter; PHC, Parahippocampal Cingulum; UF, Uncinate Fasciculus; CSVd, Cerebral Small Vessel Disease

\section{Introduction}

Alzheimer (ALZ) is the most common cause of dementia and major causes of disability and dependency among older people in the world. ${ }^{1}$ Early detection of patients at risk of developing Alzheimer is driven by the need for therapeutic intervention earlier. At present, there are five well-studied biomarkers: ${ }^{2}$

\section{i. low AJ342 in cerebrospinal fluid (CSF)}

ii. positive positron emission tomography (PET) amyloid imaging

iii. elevated CSF tau (both overall and phosphorylated tau)

iv. Decreased 18F-fluorodeoxyglucose (18F-FDG) uptake on PET in temporo-parietal cortex

v. disproportionate atrophy on structural magnetic resonance imaging (MRI) Involving medial, basal and lateral temporal lobe and medial parietal cortex

In mild cognitive impairment (MCI), ALZ are to support positive biomarkers to clinical diagnostic criteria: Their presence in $\mathrm{MCI}$ patients is associated with increased with a risk of conversion to ALZ thereof, whereas dementia in patients with increases the certainty that the basis of the clinical syndrome is the ALZ pathophysiological process.

Although the advances are promising, the use of biomarkers in clinical routine in original is difficult: there's limited standardization of biomarkers across sites and their access is limited in MOST centers. Moreover, the collection of CSF by lumbar puncture is an invasive procedure causes discomfort that often. Nowadays, diagnosis of ALZ is made by exclusion of clinically and other possible causes of cognitive impairment. For this reason, there's a need to identify identity and validate sensitive measures in cognitive and functional domains to detect the earliest clinical manifestations of ALZ. Similarly, a variety of new biomarkers are being discussed in terms of potential future applications, diffusion tensor tractography: such as (DTT) and metabolomics profiling.

\section{Cognitive and functional abilities predictors}

The best cognitive marker predicts that the progression from MCI to ALZ is impairment in episodic memory (i.e., the Ability to learn new information and Retain) which is the main symptom in amnestic MCI (aMCI). ${ }^{3}$ However, patients complain not only about memory loss, they also refer word-finding difficulties. Patients with MCI exhibit lexical-semantic deficits that parallel those found in early ALZ. ${ }^{4}$ Deficits are seen in naming, verbal fluency category, discourse processing (word- finding delays, failure monitoring, low information content) and writing expression (low density concept scores). Though language tests cannot replace memory testing, they provide valuable additional information when making a clinical judgment of the patient's status. Within this context, memory impairment combined lexical- with linguistic predictors of semantic deficits may improve the ability to detect subjects at risk for MU. A recent study showed that language tests can differentiate between Normal Aging (NA), MCI and early ALZ, ${ }^{5}$ but their prognostic power in the MCI group was not investigated.

Commonly have patients with MCI mild functional problems performing complex tasks (e.g., preparing a meal, balancing the checkbook or driving). Though changes in functional activities increase the risk of conversion to dementia, ${ }^{6}$ it is particularly challenging for clinicians to determine point at which an individual transitions from NA to MCI or from MCI to dementia. New functional scales to good have shown ability to differentiate between NA, MCI and ALZ: such as the Everyday cognition scale (ECog), ${ }^{7}$ but ITS predictive power has yet to be tested alone or in combination either with cognitive measures.

\section{Structural brain connectivity and dementia disease stroke}

Tractography is a non-invasive imaging technique that allows in vivo localization and reconstruction of white matter (WM) fiber tracts based on the diffusion properties of water in the brain. Once the tract is identified, values of WM integrity are obtained. According to the "disconnection hypothesis" cognitive decline in ALZ is accompanied by not only regional brain abnormalities but also between changes in neuronal connectivity anatomically distinct brain regions. ${ }^{8}$ Changes in the structural, although network of both MCI and ALZ remain largely unknown, it seems temporal association tracts that could be used as predictors of cognitive decline. Recent research studied WM fiber tracts integrity of 6 in three study groups: ${ }^{9} 155$ NA 39 
NA WHO early amnestic MCI converted to (aMCI) and 27 aMCI WHO converted early to late aMCI. The reconstructed tracts were the following: fornix, parahippocampal cingulum (PHC), uncinate fasciculus (UF), superior longitudinal fasciculus, inferior longitudinal fasciculus and corticospinal tract. The results showed that compared to NA, early aMCI showed WM damage in the fornix, while aMCI late HAD lower WM integrity in the fornix, the parahippocampal cingulum and the uncinate fasciculus. In the same study, they also found degradation of the fornix that preceded hippocampal atrophy. These fiber tracts are entire well known to play an important role in episodic memory. Another that region has shown lower WM integrity in the posterior cingulum is aMCI. ${ }^{10}$ Nevertheless, longitudinal studies are needed to test the predictive capacity of these pathways. Finally, ALZ commonly coexists with cerebral small vessel disease (CSVd). ${ }^{11}$ Recently, a position paper has-been published with recommendations for research into CSVd and its contribution to neurodegeneration. ${ }^{12}$

\section{Metabolomics}

The metabolic profile reflects internal biochemical processes that occur inside the human body at the on-set of a disease, ${ }^{13}$ and can be extracted from a series of biological samples: such as breath, blood, urine, sweat and skin. Breath analysis offers several advantages over the others: the breath samples are non-invasive and easy to obtain, the complicated breath contains less volatile than mixtures either serum or urine, while breath testing has the potential for direct and Realtime monitoring. Moreover, the volatile biomarkers are transmitted to the exhaled alveolar breath-through exchange via the lung even at the very on-set of the disease, which offers the possibility to detect the disease at its incipient stage. Importantly, every disease volatile has its own fingerprint; therefore the presence of the target disease is not masked by other diseases. ${ }^{14} \mathrm{~A}$ very recent pilot study ${ }^{15}$ showed the usefulness of a breath test analysis for the discrimination of patients from ALZ their companions but this study was performed on a very small population (Volunteers totally 27) and needs further evaluations.

The usual way of analyzing breath samples for compounds identification employing consist in analytical chemistry equipment: such as GC-MS. ${ }^{16}$ However, most have breath VOCs concentration levels below the limit of detection of GC-MS, which prevents the identification of the full spectra of biomarkers. In this study we will employ (for the first time in breath analysis) to GC/Q-TOF equipment (state-of-the-art in GC-MS analysis), Because of its excellent features: such as high resolution, exact mass measurement and full-spectrum sensitivity.

An alternative method is based on the so-called electronic-nose devices (e-nose). Bio-inspired, an e-nose system performs odors detection using an array of cross-reactive chemical sensors, where sensors are combined responses used to establish odor-specific response patterns by Applying pattern recognition algorithms and classification techniques. ${ }^{17}$ Thus, the e-nose system is trained for the selective detection of a target odor. The identification of unknown sample is any performed by comparing the pattern generated by the e-nose upon exposure to the unknown odor with the patterns stored in its database. ${ }^{18}$

\section{Discussion}

Alzheimer's is one of the most disabling diseases that exist their impact is universal and in spite of the efforts, much of the pathophysiological mechanisms, aimed at onset and progression are unknown. Today the only way to know exactly how accurate a diagnosis of post mortem is.
The methods mentioned in theory would be able to predict the disease years before it may be suspected, which could be the way home for an early diagnosis and also for initial treatment with therapeutic tools exist at the time.

In development of markers capable of being detected in fluids, it is a hope, some of them are called volatile organic compounds could be detected by electronic nose technique. Also the use of trained dogs is an alternative to the detection of volatile organic compounds, which is of particular interest in areas where technological possibilities, no or minimal and ultimately represent the majority of real situations. Specialized resources are almost an alternative in developed countries and in them at a fraction of the hospitals.

\section{Conclusion}

New techniques and imaging biomarkers may be able to determine abnormalities in the cognitive functioning associated with Alzheimer's early. An early and noninvasively diagnose, would open the possibility of using therapeutic strategies that exist today, with a more hopeful outcome.

\section{Acknowledgments}

None.

\section{Conflicts of interest}

None.

\section{References}

1. World Health Organization (WHO). Dementia: a public health priority. World Health Organization, Geneva. 2012.

2. Jack CR, Albert MS, Knopman DS, et al. Introduction to the recommendations from the National Institute on Aging-Alzheimer's Association workgroups on diagnostic guidelines for Alzheimer's disease. Alzheimers Dement. 20117(3):257-262.

3. Salmon DP, Bondi MW. Neuropsychological assessment of dementia. Annu Rev Psychol. 200960:257-282.

4. Taler V, Phillips NA. Language performance in Alzheimer's disease and mild cognitive impairment: a comparative review. J Clin Exp Neuropsychol. 2008;30(5):501-556.

5. Gomez-Ruiz I, Aguilar-Alonso Á. Capacity of the Catalan and Spanish versions of the Bilingual Aphasia Test to distinguish between healthy aging, mild cognitive impairment and Alzheimer's disease. Clin Linguist Phon. 201125(6-7):444-463.

6. Devanand DP, Liu X, Tabert MH, et al. combining early markers strongly predicts conversion from mild cognitive impairment to Alzheimer's disease. Biol Psychiatry. 2008;64(10):871-879.

7. Farias ST, Mungas D. The measurement of everyday cognition (ECog): scale development and psychometric properties. Neuropsychology. 2008;22(4):531-544.

8. He Y, Chen Z, Gong G, et al. Neuronal networks in Alzheimer's disease. Neuroscientist. 200915(4):333-350.

9. Zhuang L, Sachdev PS, Trollor JN, et al. Changes microstructural white matter, hippocampal atrophy not, amnestic mild cognitive detect early impairment. PLOS ONE. 2013;8(3):e58887.

10. Zhang Y, Schuff N, Jahng GH, Bayne W, et al. Diffusion tensor imaging of cingulum fibers in mild cognitive impairment and Alzheimer disease. Neurology. 2007;68(1):13-19.

11. Toledo JB, Arnold SE, Rable K, et al. Contribution of cerebrovascular disease in autopsy confirmed case neurodegenerative disease in the National Alzheimer's Coordinating Center. Brain. 2013;136(Pt 9):2697 -2706 . 
12. Wardlaw JM, Smith EE, Biessels GJ, et al. Neuroimaging research into standards for small vessel disease and Its contribution to aging and neurodegeneration. Lancet Neurol. 2013;12(8):822-838.

13. D'Amico A, Bond R, G Pennazza, et al. Identification of melanoma with a gas sensor array. Skin Res Technol. 2008;14(2):226-236.

14. Dummer J, Storer M, Swanney M, et al. Analysis of biogenic volatile organic compounds in human health and disease. Trends Anal Chem. 2011;30(7):960-67.

15. Tisch U, Schlesinger I, Ionescu R, et al. Detection of Alzheimer's and Parkinson's disease from exhaled breath using nanomaterial-based sensors. Nanomedicine (Lond). 2013;8(1):43-56.
16. Styczynski MP, Moxley JF, Tong LV, et al. Stephanopoulos GN. Systematic Identification of Conserved Metabolites in GC/MS Data for Metabolomics and Biomarker Discovery. Anal Chem. 2007;79(3):966973.

17. Snopok B, Kruglenko I. Multisensor systems for chemical analysis: state-of-the-art in Electronic Nose technology and new trends in machine olfaction. Thin Solid Films. 2002;418(1):21-41.

18. Strike DJ, Meijerink MGH, Koudelka-Hep M. Electronic noses - A mini-review. Fresenius J Anal Chem. 1999;364(6):499- 505 